\title{
Identification of parental line specific effects of MLF2 on resistance to coccidiosis in chickens
}

\author{
Yeong Ho Hong ${ }^{1}$, Eui-Soo Kim² ${ }^{2}$ Hyun S Lillehoj ${ }^{3^{*}}$ \\ From International Symposium on Animal Genomics for Animal Health (AGAH 2010) \\ Paris, France. 31 May - 2 June 2010
}

\begin{abstract}
Background: MLF2 was the candidate gene associated with coccidiosis resistance in chickens. Although single marker analysis supported the association between MLF2 and coccidiosis resistance, causative mutation relevant to coccidiosis was not identified yet. Thus, this study suggested segregation analysis of MLF2 haplotype and the association test of the other candidate genes using improved data transformation.

Results: A haplotype probably originated from one parental line was found out of 4 major haplotypes of MLF2. Frequency of this haplotype was 0.2 in parental chickens and its offspring in 12 families. Allele substitution effect of the MLF2 haplotype originated from a specific line was associated with increased body weight and fecal egg count explaining coccidiosis resistance. Nevertheless Box-Cox transformation was able to improve normality; association test did not produce obvious different results compared with analysis with log transformed phenotype.

Conclusion: Allele substitution effect analysis and classification of MLF2 haplotype identified the segregation of haplotype associated with coccidiosis resistance. The haplotype originated from a specific parental line was associated with improving disease resistance. Estimating effect of MLF2 haplotype on coccidiosis resistance will provide useful information for selecting animals or lines for future study.
\end{abstract}

\section{Background}

Avian coccidiosis impairs the growth and feed efficiency of infected chickens [1]. There were evidences that resistance to avian coccidiosis is associated with inheritance and coccidiosis resistant line could be established by selection [2]. The resistance to avian coccidiosis QTL has been identified near two microsatellite markers on chromosome $1[3,4]$. Associations between parameters of resistance to coccidiosis and single nucleotide polymorphisms (SNPs) in 3 candidate genes located around QTL on chromosome 1 (Zyxin, TCR- $\beta, M L F 2$ ) were determined [5,6]. These studies showed the SNPs in $M L F 2$ are the most probable locus associated with coccidiosis resistance in chickens. The current analysis was conducted to identify the parental line specific haplotype of $M L F 2$. Although $M L F 2$ explained variation of body

\footnotetext{
* Correspondence: Hyun.Lillehoj@ars.usda.gov

${ }^{3}$ Animal Parasitic Diseases Laboratory, Animal and Natural Resources Institute, United States Department of Agriculture, Beltsville, MD 20705, USA Full list of author information is available at the end of the article
}

weight affected by coccidiosis, the association of causative mutation was not obviously identified [6]. Thus, this study was suggested to identify segregation of $M L F 2$ allele associated with coccidiosis resistance for practical application and future study for identifying causative mutation. Since oocyst shedding significantly deviated from normal distribution, transformation method may affect association test. In previous studies, oocyst shedding was transformed using log transformation, but normality was fully satisfied $(p \sim 0.01)$ by log transformation compared with Box-Cox transformation ( $p>$ 0.05). Box-Cox transformation of oocyst shedding was used to evaluate association between oocyst shedding and SNPs, including candidate genes analyzed in previous studies.

\section{Methods}

Chickens from coccidiosis resistant and susceptible commercial broiler lines were crossed to produce $F_{1}$ chickens [3]. Twelve pairs of $F_{1}$ individuals were mated 
to produce $290 \mathrm{~F}_{2}$ offspring from four hatches. $\mathrm{F}_{2}$ chickens were orally infected with $1.0 \times 10^{4}$ of sporulated $E$. maxima oocysts at 4 weeks of age [3]. Body weight was measured on days 3, 6, and 9 post-infection (PI) as previously described $[7,8]$. The number of oocysts was evaluated using the fecal sample collected between Days 5 to 9 PI by method of Lillehoj and Ruff (1987).

Genomic DNA was extracted from erythrocytes using the GenElute Blood Genomic DNA kit (Sigma, St. Louis, MO). PCR product was sequenced with internal primers using an ABI 3730 DNA analyzer (Applied Biosystem, Foster City, CA). SNP was genotyped by genomic DNA sequencing with the same gene-specific internal primers $[5,6]$. Then, haplotype phase was decided based on pedigree information. Associations between SNP genotypes or haplotypes and disease phenotypes were evaluated using linear model including family, sex, and hatch effects [5]. The number of oocyst was transformed by log transformation, and it was also transformed using an empirical approach suggested by Box-Cox [9]. These methods use the profile likelihood function for the largest linear model to be considered as a guide in choosing a value for parameter [10].

\section{Results}

Ten SNPs were identified in the zyxin, 12 in the TCR- $\beta$ and 4 in the MLF2 gene [5,6]. At various times following experimental infection of the $\mathrm{F}_{2}$ generation with Eimeria maxima, body weights, fecal oocyst shedding, and biochemical parameters were measured as parameters of coccidiosis resistance [3]. Single marker and haplotype-based tests were applied to determine the associations between SNPs and the parameters of coccidiosis resistance including body weight and Box-Cox transformed oocyst shedding. The maximum additive genetic effect on disease resistance of a SNP in $M L F 2$ was explained by body weight $(p=0.0002)$ and this SNP of $M L F 2$ also significantly associated with body weight was also associated with fecal oocyst shedding [6]. This was confirmed using Box-Cox transformed oocyst shedding data in this study. Box-Cox transformed fecal oocyst shedding was close to normal distribution (Kolmogorov-Smirnov normality test, $p>0.1$ ) compared with $\log$ transformed data $(p>0.01)$. Associations between Box-Cox transformed oocyst shedding number and SNP was summarized in Table 1. This analysis support evidence of association between markers in $M L F 2$ gene and oocyst shedding. Association between transformed oocyst shedding and the other genes including zyxin /TCR- $\beta$ were also detected $(p<0.05)$ using BoxCox transformed oocyst shedding, which was not significant using log transformed data. The significance level of association between the other markers and oocyst shedding was not changed obviously at various
Table 1 Association test using oocyst shedding transformed by Box-Cox transformation and log transformation

\begin{tabular}{ccc}
\hline SNP & Log transformation & Box-Cox transformation \\
\hline${ }^{1}$ zyxin SNP_187 & 0.08 & 0.04 \\
${ }^{2}$ TCR- $\beta$ SNP_177 & 0.06 & 0.02 \\
${ }^{2}$ MLF2-SNP_892 & 0.002 & 0.001 \\
${ }^{2}$ MLF2-SNP_947 & 0.02 & 0.02 \\
\hline
\end{tabular}
${ }^{1}[5]$.

${ }^{2}[6]$.

thresholds ( $p=0.05,0.01$ or 0.001 ) compared with analysis using log transformed oocyst shedding.

Four haplotypes accounted for $98 \%$ of all observed MLF2 SNP (Table 2). The haplotype association test was used to determine the relationship between the oocyst shedding number and the haplotypes of the $M L F 2$. The allele substitution effect of $M L F 2$ haplotype 4 versus haplotypes 1 and 3 was significantly associated with increased body weight and oocyst shedding number (Table 2). The MLF2 homozygous haplotypes $2 / 2$ and $4 /$ 4 were found in 2 and 4 animals, respectively (Tables 3 , 4). However, homozygous haplotypes 1 and 3 were not observed in 24 parent chickens, which implies that only haplotypes $1(n=4)$ and $3(n=10)$ were possibly originated from a specific parental chicken line. The haplotype substitution effect of haplotype 3 was higher than allele substitution effect of haplotypes 1 and 2 (Tables 3, 4). Haplotypes 2 and 4 were found in both parental line, but haplotypes 1 and 3 were likely to be originated from specific parental line. However, haplotypes 1 and 3 were likely to be originated from different parental chicken lines considering high heterozygous frequency of haplotypes 1 and 3 (Table 3 ).

\section{Conclusions}

Single marker association analysis for 16 traits of coccidiosis resistance showed SNPs of TCR- $\beta$ and $M L F 2$ were associated with oocyst shedding and body weights in previous study [6], and it was confirmed by additional association tests in current study. In this study allele substitution effect analysis and classification of $M L F 2$ haplotype elucidated parental origin of haplotype

Table 2 Allele (haplotype) substation effect of MLF2

\begin{tabular}{lcccc}
\hline Haplotype & ID & Frequency & $\begin{array}{c}\text { Body weight } \\
\text { (s.d.) }\end{array}$ & $\begin{array}{c}\text { Oocyst shedding } \\
\text { (s.d.) }\end{array}$ \\
\hline A A T A & 1 & 0.12 & $88.8(42.1)^{* *}$ & $2.63(0.05)^{*}$ \\
A T T A & 2 & 0.26 & $41.7(31.4)$ & $1.47(0.17)$ \\
A T T G & 3 & 0.20 & $112.0(38.0)^{* *}$ & $2.13(0.07)^{+}$ \\
G T T A & 4 & 0.41 & - & - \\
others & - & 0.01 & - & - \\
\hline
\end{tabular}

** $p<0.01,{ }^{*} p<0.05,+p<0.1$ 
Table 3 MLF2 haplotype in 24 parental chickens (F1)

\begin{tabular}{cccccc}
\hline Haplotype* $^{*}$ id & Number in parents & Homozygote & Heterozygous with haplotype $\mathbf{3}$ & Origin of haplotype \\
\hline A T T A & 1 & 4 & 0 & 2 & one or both \\
A A T A & 2 & 14 & 2 & 4 & both \\
A T T G & 3 & 10 & 0 & 3 & one or both \\
G T T A & 4 & 18 & 4 & 1 & both \\
others & - & 2 & 0 & na \\
\hline
\end{tabular}

*[6]

associated with coccidiosis resistance. The fourth [A/G] SNP in $M L F 2$ was major determinant of haplotype 3 vs the other haplotypes, but allele A of the first SNP [A/G] was common in haplotypes 1, 2 and 3. Haplotype association test was applied to estimate effect of each haplotype which was not revealed clearly by single marker association tests. Difference between additive effect (body weight and oocyst) of haplotypes 1 and 3 was smaller than that of haplotypes 2 and 3. However, haplotypes 1 and 3 are likely to be originated from the different parental lines. In genome-wide [3] and fine mapping [4] of coccidiosis resistance, QTL was not detected in some chicken families. These previous linkage QTL mapping studies could be affected by heterogeneity in parental lines. The haplotype (ATTG) of MLF2

Table 4 Haplotypes of MLF2 in 24 parental chickens

\begin{tabular}{cccccc}
\hline Faimly-ID & $\mathbf{1}$ & $\mathbf{2}$ & $\mathbf{3}$ & $\mathbf{4}$ & others \\
\hline F1-1 & 1 & 1 & 0 & 0 & 0 \\
F1-2 & 1 & 0 & 0 & 1 & 0 \\
F2-1 & 1 & 0 & 1 & 0 & 0 \\
F2-2 & 0 & 0 & 1 & 1 & 0 \\
F3-1 & 0 & 1 & 0 & 1 & 0 \\
F3-2 & 0 & 0 & 0 & 1 & 1 \\
F4-1 & 0 & 1 & 1 & 0 & 0 \\
F4-2 & 0 & 1 & 1 & 0 & 0 \\
F5-1 & 0 & 1 & 0 & 1 & 0 \\
F5-2 & 0 & 1 & 0 & 1 & 0 \\
F6-1 & 0 & 0 & 1 & 1 & 0 \\
F6-2 & 0 & 0 & 0 & $\mathbf{2}$ & 0 \\
F7-1 & 0 & $\mathbf{2}$ & 0 & 0 & 0 \\
F7-2 & 0 & 1 & 1 & 0 & 0 \\
F8-1 & 0 & 1 & 0 & 1 & 0 \\
F8-2 & 0 & 0 & 0 & $\mathbf{2}$ & 0 \\
F9-1 & 0 & 0 & 1 & 0 & 1 \\
F9-2 & 0 & 0 & 0 & $\mathbf{2}$ & 0 \\
F10-1 & 0 & 1 & 0 & 1 & 0 \\
F10-2 & 0 & 0 & 1 & 1 & 0 \\
F11-1 & 0 & 1 & 1 & 0 & 0 \\
F11-2 & 0 & 0 & 0 & $\mathbf{2}$ & 0 \\
F12-1 & 0 & $\mathbf{2}$ & 0 & 0 & 0 \\
F12-2 & 1 & 0 & 1 & 0 & 0 \\
\hline
\end{tabular}

originated from a specific parental line was associated with elevated body weight and oocyst shedding number. Identifying haplotype diversity of $M L F 2$ in other chicken families will provide useful information for experimental designs in the future studies.

List of abbreviations used

SNPs: single nucleotide polymorphisms; MLF2: Myeloid Leukemia Factor 2

\section{Acknowledgments}

This Project was supported, in part, by the National Research Initiative of the Cooperative State Research, Education and Extension Service at USDA (NRI grant \#2004-35204-14798).

This article has been published as part of BMC Proceedings Volume 5 Supplement 4, 2011: Proceedings of the International Symposium on Animal Genomics for Animal Health (AGAH 2010). The full contents of the supplement are available online at http://www.biomedcentral.com/1753$6561 / 5$ ? issue $=\$ 4$

\section{Author details}

${ }^{1}$ Department of Animal Science and Technology, Chung-Ang University Gyeonggi-Do 456-756, Korea. ${ }^{2}$ Bovine Functional Genomics Laboratory, Animal and Natural Resources Institute, United States Department of Agriculture, Beltsville, MD 20705, USA. ${ }^{3}$ Animal Parasitic Diseases Laboratory, Animal and Natural Resources Institute, United States Department of Agriculture, Beltsville, MD 20705, USA.

\section{Authors' contribution}

$\mathrm{YHH}$; genotyping and manuscript preparation, ESK; genetic analysis, HSL; grant preparation and experiment design

\section{Competing interests}

The authors declare that they have no competing interests.

Published: 3 June 2011

\section{References}

1. Lillehoj HS, Kim CH, Keeler CL, Zhang S: Immunogenomic approaches to study host immunity to enteric pathogens. Poultry Science 2007, 86(7):1491-1500.

2. Johnson LW, Edgar SA: Response to prolonged selection for resistance and susceptibility to acute cecal occidiosis in the Auburn strain Single Comb White Leghorn. Poult Sci 1982, 61(12):2344-2355.

3. Zhu JJ, Lillehoj HS, Allen PC, Van Tassell CP, Sonstegard TS, Cheng HH, Pollock D, Sadjadi M, Min W, Emara MG: Mapping quantitative trait loci associated with resistance to coccidiosis and growth. Poultry Science 2003, 82(1):9-16.

4. Kim ES, Hong YH, Min W, Lillehoj HS: Fine-mapping of coccidia-resistant quantitative trait loci in chickens. Poultry Science 2006, 85(11):2028-2030.

5. Hong $Y H$, Kim ES, Lillehoj $H$, Song KD, Lillehoj E: Association of resistance to avian coccidiosis with single nucleotide polymorphisms in the Zyxin gene. Poultry Science 2009, 88(3):511-518.

6. Kim ES, Hong YH, Lillehoj HS: Genetic effects analysis of myeloid leukemia factor 2 and $\mathrm{T}$ cell receptor-beta on resistance to coccidiosis in chickens. Poultry Science 2010, 89(1):20-27. 
7. Allen PC: Production of free radical species during Eimeria maxima infections in chickens. Poultry Science 1997, 76(6):814-821.

8. Yun $\mathrm{CH}$, Lillehoj $\mathrm{HS}$, Zhu J, Min W: Kinetic differences in intestinal and systemic interferon- $\gamma$ and antigen-specific antibodies in chickens experimentally infected with Eimeria maxima. Avian Dis 2000, 44(2):305-312.

9. Box GEP, Cox DR: An analysis of transformations (with discussion). Journal of the Royal Statistical Society B 1964, 26:211-252.

10. Venables WN, Ripley BD: Modern applied statistics with S-plus. Springer; 1999.

doi:10.1186/1753-6561-5-S4-S21

Cite this article as: Hong et al:: Identification of parental line specific effects of MLF2 on resistance to coccidiosis in chickens. BMC Proceedings 2011 5(Suppl 4):S21.

Submit your next manuscript to BioMed Central and take full advantage of:

- Convenient online submission

- Thorough peer review

- No space constraints or color figure charges

- Immediate publication on acceptance

- Inclusion in PubMed, CAS, Scopus and Google Scholar

- Research which is freely available for redistribution

Submit your manuscript at www.biomedcentral.com/submit 